\title{
Anorectal functional outcome following laparoscopic low anterior resection for rectal cancer
}

\author{
LI-GUO LIU $^{1 *}$, XUE-BING YAN $^{1 *}$, ZE-ZHI SHAN $^{1}$, LEI-LEI YAN ${ }^{1}$, \\ CHUN-YU JIANG ${ }^{2}$, JIA ZHOU ${ }^{2}$, YUAN TIAN ${ }^{1}$ and ZHI-MING JIN ${ }^{1}$ \\ Departments of ${ }^{1}$ Surgery and ${ }^{2}$ Radiology, Sixth People's Hospital Affiliated to \\ Shanghai Jiao Tong University, Shanghai 200233, P.R. China
}

Received August 19, 2016; Accepted January 23, 2017

DOI: $10.3892 / \mathrm{mco} .2017 .1183$

\begin{abstract}
Low anterior resection (LAR) with total mesorectal excision has been considered a standard treatment for patients with rectal cancer. However, the functional outcome and life quality of laparoscopic LAR (LLAR) in Chinese patients remain unclear. A cohort of 51 Chinese patients (22 men and 29 women) who had undergone LLAR was included in this study. Anorectal manometry combined with the Wexner scores questionnaire were applied to assess functional outcome preoperatively (1 week) and postoperatively (at 3, 6 and 9 months). The validated Chinese versions of the European Organization for Research and Treatment of Cancer QLQ-C30 and QLQ-CR38 questionnaires were also used to assess the patients' quality of life at the indicated time points. The results demonstrated that the manometric parameters exhibited a temporary decrease at 3 months postoperatively, but a gradual increase at 6 and 9 months, while the Wexner scores exhibited an opposite trend. Furthermore, patients with high anastomoses had significantly higher manometric parameters, a lower frequency of incontinence and lower Wexner scores compared with those with low anastomoses at 9 months (all $\mathrm{P}<0.05$ ). For the entire cohort, quality of life at 3 months postoperatively was worse compared with the preoperative level, but returned to normal by 9 months. Patients with high anastomoses exhibited significantly better role, emotional and social function, had a better body image and sexual function, fewer problems with defecation and lower frequency of diarrhea, as well as fewer chemotherapy-related side effects at 6 months postoperatively when compared with the low anastomosis group (all
\end{abstract}

Correspondence to: Dr Zhi-Ming Jin, Department of Surgery, Sixth People's Hospital Affiliated to Shanghai Jiao Tong University, 600 Yishan Road, Shanghai 200233, P.R. China

E-mail: jzmgyp@aliyun.com

*Contributed equally

Key words: rectal cancer, laparoscopic low anterior resection, anorectal function, manometry
$\mathrm{P}<0.05)$. In conclusion, LLAR is generally acceptable for Chinese patients with rectal cancer, particularly for those with middle or high rectal cancer, in terms of functional outcome and quality of life.

\section{Introduction}

Colorectal cancer (CRC) is one of the most commonly diagnosed cancers worldwide, with an overall incidence rate of 188.4/100,000 and a mortality rate of 120.1/100,000 in Eastern Asia (1). Approximately one-third of all CRCs are localized in the rectum (2). In China, CRC was ranked the fourth most common type of cancer, which may be attributed to the changes in lifestyle (3).

Despite encouraging advances in chemoradiotherapy and targeted therapy, surgery remains the cornerstone of treatment for rectal cancer. The surgical treatment for rectal cancer is usually a challenge for a number of surgeons, as they must consider the balance between cancer curability and functional preservation. In the past, abdominoperineal resection (APR) with the construction of a permanent stoma was the standard treatment for rectal cancer. However, this surgical technique inevitably has an adverse impact on patients' quality of life (QoL) (4). Subsequently, with the rapid technical improvements, a sphincter-preserving procedure, referred to as low anterior resection (LAR), has become the preferred method for patients with rectal cancer and previous studies have suggested that LAR has a similar oncological efficacy but a more favorable outcome in terms of the patients' postoperative QoL compared with APR (5-8). Laparoscopic surgery as an emerging technique, has been recently proven to be a feasible alternative to open surgery for rectal cancer in terms of oncological outcome. However, it is suggested to be superior to traditional open surgery, largely due to its favorable clinical outcomes, including reduced blood loss, less pain and shorter recovery time (9). Therefore, laparoscopic LAR (LLAR) is widely used for patients with rectal cancer. Numerous studies have attempted to investigate its effect on patient outcome, in terms of oncological results or QoL evaluation, but without taking the significance of anorectal functional outcome into consideration $(10,11)$.

Anorectal function is a key factor affecting the patients' QoL and current studies evaluating this function are mostly 
based on subjective questionnaires, which may be easily influenced by various social/individual factors, and may not accurately reflect the postoperative anorectal function (12-14). Therefore, a novel objective method combined with the traditional questionnaire survey should be optimally applied to evaluate the change in anorectal function following rectal surgery. Anorectal manometry has been found to be an effective and objective test for evaluating postoperative anorectal function $(15,16)$. We previously performed anorectal manometry combined with a questionnaire survey to evaluate the anorectal function of Chinese patients following partial intersphincteric resection and transanal endoscopic microsurgery (17-19). However, to the best of our knowledge, only a limited number of studies employing manometry to evaluate anorectal function following LLAR are available, and the number is even lower for Chinese patients. Therefore, the aim of the present study was to evaluate the anorectal function of Chinese patients following LLAR using anorectal manometry and the Wexner questionnaire survey. To further validate the postoperative effect of LLAR on patient QoL, the acknowledged QLQ-C30 and QLQ-CR38 questionnaires were also employed.

\section{Materials and methods}

Patient data. Between January, 2013 and December, 2014, a cohort of 51 patients ( 22 men and 29 women), with a median age of 52 years (range, 36-75 years) who had undergone LLAR at the Department of General Surgery, Sixth People's Hospital Affiliated to Shanghai Jiao Tong University (Shanghai, China) were included in our study. All the patients were clinicopathologically diagnosed with adenocarcinoma of the rectum (within $5-12 \mathrm{~cm}$ above the anal verge). Tumor location was assessed by preoperative examination, including digital rectal examination and colonoscopy. In addition, the postoperative anastomotic height (measured from the anastomosis to the anal verge) was assessed by colonoscopy and the patients were allocated into two groups: The high anastomosis (HA) group, comprising those with anastomosis $>5 \mathrm{~cm}$ above the anal verge, and the low anastomosis (LA) group, comprising those who had an anastomosis within $5 \mathrm{~cm}$ of the anal verge. There was no significant difference in general information between the two groups (all $\mathrm{P}>0.05$ ) as shown in Table I. The exclusion criteria were applied as follows: i) Abdominal computed tomography and magnetic resonance imaging showing distal metastases or local invasion into the levator ani muscle and the external sphincter; ii) laparoscopic surgery converted to open surgery due to unexpected factors; and iii) patients with accompanying symptoms including intestinal obstruction or perforation, dementia and cognitive dysfunction. Furthermore, another cohort of 50 patients (32 men and 18 women), with a median age of 51 years (range, 32-72 years) who had undergone other abdominal surgeries (not involving the pelvis) were also included in our study as the control group to test the consistency of the baseline and reduce bias. All the patients received the same chemotherapy (12 courses of FOLFOX4) postoperatively and patients who received radiotherapy were not included in our study. The study was approved by the Ethics Committee of The Sixth People's Hospital Affiliated to Shanghai Jiao Tong University.
Written informed consent was obtained from all the patients prior to enrolment.

Surgical technique. All the surgical procedures were conducted by the same group of surgeons experienced in laparoscopic colorectal surgery; bowel preparation and perioperative intravenous antibiotic prophylaxis were routinely performed. The laparoscopic process for tumor resection has been previously described (20). In brief, after establishing a pneumoperitoneum, a sharp dissection involving a total mesorectal excision was performed by an ultrasound knife. The inferior mesenteric vessels were ligated following identification of the left ureter, and the distal rectum was successively separated. The proximal end of the bowel was delivered through a small incision. Finally, the bowel was resected with a distal mesorectal margin of $\geq 2 \mathrm{~cm}$ whenever possible, and the double-stapling technique was used to perform an anastomosis as described by Kosmidis et al (21). The presence of an anastomotic leak was checked by transrectal insufflation of air. For postoperative oncological follow-up, the patients underwent laboratory tests every 3 months and radiological examination every 6 months to monitor local recurrence and/or distal metastasis. Endoanal ultrasonography was also employed to investigate the integrity and thickness of the internal anal sphincter (IAS) and the external anal sphincter (EAS) postoperatively.

Functional assessment. For anorectal manometry, an 8-channel water-perfused catheter with an external diameter of $5.5 \mathrm{~mm}$ and a computer system (all from Medical Measurement Systems Corporation, Enschede, Netherlands) were employed. Each patient assumed the left lateral decubitus position and the stationary technique was used for catheter insertion. Anorectal manometric parameters, including mean/maximal anal resting pressure (mean/max ARP), maximal squeeze pressure (MSP), initial/strong sensory volume (ISV/SSV), maximal tolerable volume (MTV) and rectoanal inhibitory reflexes (RAIR) were recorded. In a proportion of patients with low anastomosis, postoperative manometry performed within a short period may result in rupture of the anastomosis. Therefore, manometry was performed at 1 week preoperatively and at 3,6 and 9 months postoperatively.

Questionnaire assessment for anal function and life quality. The anal function was assessed based on Wexner incontinence grading scale, while QoL was assessed by the QLQ-C30 and QLQ-CR38 questionnaires developed by the European Organisation for Research and Treatment of Cancer. The Chinese versions of QLQ-C30 and QLQ-CR38, the clinical validity and reliability of which have been previously confirmed, were used for the questionnaire survey $(10,22)$. For this questionnaire survey, all the patients were interviewed with standardized questionnaires at 1 week preoperatively and at 3,6 and 9 months postoperatively.

Statistical analysis. The results are presented as mean \pm standard deviation (SD). The Student's $t$-test and the $\chi^{2}$ test were performed by SPSS 17.0 statistical software (SPSS, Inc, Chicago, IL, USA). A P-value of $<0.05$ was considered to indicate statistically significant differences. 
Table I. Clinicopathological characteristics of rectal cancer patients following laparoscopic low anterior resection.

\begin{tabular}{lcc}
\hline Characteristics & HA group & LA group \\
\hline Number of patients & 31 & 19 \\
Age, years (mean \pm SD) & $50.6 \pm 13.3$ & $52.7 \pm 14.7$ \\
Gender (male/female) & $14 / 17$ & $7 / 12$ \\
Body mass index, $\mathrm{kg} / \mathrm{m}^{2}$ (mean \pm SD) & $21.8 \pm 4.4$ & $21.6 \pm 4.9$ \\
Number of patients with comorbidities & 0 & 0 \\
Marital status (married/divorced/widowed/single) & $29 / 2 / 0 / 0$ & $18 / 1 / 0 / 0$ \\
Education level (underprimary/primary/ & $8 / 7 / 10 / 6$ & $5 / 4 / 7 / 3$ \\
secondary/tertiary or higher) & & 0 \\
Number of patients with complications & 0 & 0 \\
Reoperation & 0 & $3 / 16$ \\
Tumor stage (II/III) & $5 / 26$ &
\end{tabular}

HA, high anastomosis; LA, low anastomosis; SD, standard deviation.

\section{Results}

General description. All the patients were followed up for at least 1 year and there were no reported deaths. Furthermore, radiological examination indicated no local recurrence or distant metastasis. Four patients had anastomotic leakage and received conservative treatment. One patient of the LA group complained of severe stool incontinence and his manometric parameters were by $50 \%$ lower compared with those of other patients at 9 months postoperatively. Therefore, this case was not included in our data.

Changes in anorectal pressure and volume. First, as shown in Table II, there was no significant difference in the preoperative pressure between the control and trial groups (all $\mathrm{P}>0.05$ ). Generally, all the manometric parameters changed to different degrees following LLAR (Fig. 1A-C). For the HA group, the mean/maximum ARP and MSP were decreased at 3 months postoperatively (all $\mathrm{P}<0.05$ ), but had returned to preoperative levels at 6 months postoperatively (all P>0.05). For the LA group, the observations were similar at 3 months postoperatively (all $\mathrm{P}<0.05$ ), but the parameters gradually returned to normal at 9 months postoperatively $(\mathrm{P}>0.05)$, except for MSP, which had returned to normal level at 6 months $(\mathrm{P}>0.05)$. With regard to intergroup comparison, the MSP of the HA group was significantly higher compared with that of the LA group at 3 months $(\mathrm{P}<0.05)$. In addition, the mean/maximal ARP of the HA group was significantly higher compared with that of the LA group at 6 months postoperatively (all $\mathrm{P}<0.05$ ).

The ISV, SSV and MTV were significantly decreased in both groups at 3 months postoperatively (all $\mathrm{P}<0.05$ ), but had gradually improved at 6 and 9 months postoperatively (Fig. 1D-F). However, all the volume parameters remained significantly lower compared with the preoperative level at 9 months postoperatively (all $\mathrm{P}<0.05$ ). Furthermore, the MTV of the HA group was significantly higher compared with that of the LA group at 9 months postoperatively $(\mathrm{P}<0.05)$.
Table II. Baseline of functional parameters at 1 week preoperatively.

\begin{tabular}{lcc}
\hline Preoperative & Control group & Trial group \\
\hline Mean ARP, mmHg & $45.1 \pm 5.4$ & $40.2 \pm 6$ \\
Maximum ARP, mmHg & $67 \pm 7.5$ & $62.3 \pm 7.2$ \\
MSP, mmHg & $184.1 \pm 20.6$ & $180.4 \pm 21.7$ \\
ISV, ml & $110.3 \pm 12.1$ & $105.4 \pm 12.4$ \\
SSV, ml & $130.2 \pm 14.3$ & $126.2 \pm 15.7$ \\
MTV, ml & $190.7 \pm 20.1$ & $180.4 \pm 21.8$ \\
RAIR present & $50(100.0)$ & $48(96.0)$ \\
IAS, mm & $2.1 \pm 0.4$ & $1.9 \pm 0.5$ \\
EAS, mm & $4 \pm 0.5$ & $3.8 \pm 0.7$ \\
Gas/liquid/solid incontinence & $0 / 0 / 0$ & $0 / 0 / 0$ \\
Wexner scores & 0 & 0 \\
\hline
\end{tabular}

Values are presented as absolute numbers, mean \pm standard deviation, or number (\%). ARP, anal resting pressure; MSP, maximal squeeze pressure; ISV, initial sensory volume; SSV, strong sensory volume; MTV, maximal tolerable volume; RAIR, rectoanal inhibitory reflex; IAS, internal anal sphincter; EAS, external anal sphincter.

Changes in rectoanal inhibitory reflex (RAIR) and anal sphincter. The changes in RAIR following LLAR are shown in Fig. 2A. Generally, RAIR was absent in 2 of the 50 patients at 1 week preoperatively. For the HA group, RAIR was absent in 12 of the 31 patients at 3 months postoperatively, whereas RAIR was absent in none of the patients at 6 and 9 months postoperatively. For the LA group, RAIR was absent in 10 of the 19 patients at 3 months postoperatively. However, RAIR was induced in 16 of the 19 patients at 6 and 9 months postoperatively, with 3 patients remaining negative for RAIR (including 2 patients who were negative for RAIR preoperatively). In addition, the RAIR rate of the HA group was significantly higher compared with that of the LA group between 3 and 9 months postoperatively 

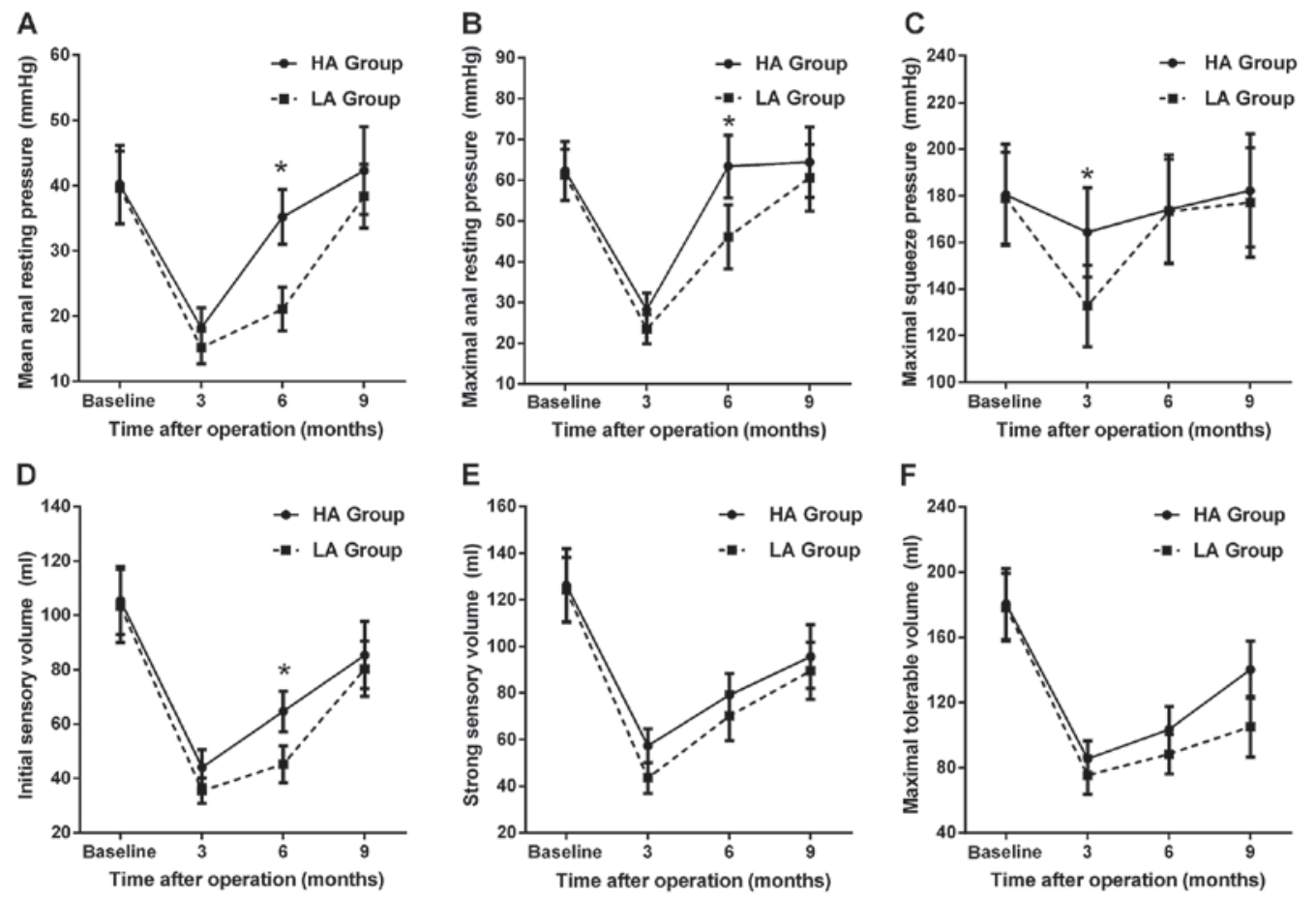

Figure 1. Anorectal manometry for anal functional assessment. (A) Mean anal resting pressure. (B) Maximal anal resting pressure. (C) Maximal squeeze pressure. (D) Initial sensory volume. (E) Strong sensory volume. (F) Maximal tolerable volume. Error bars indicate the standard error of the mean. *, statistically significant difference $(\mathrm{P}<0.05)$. HA, high anastomosis; LA, low anastomosis.
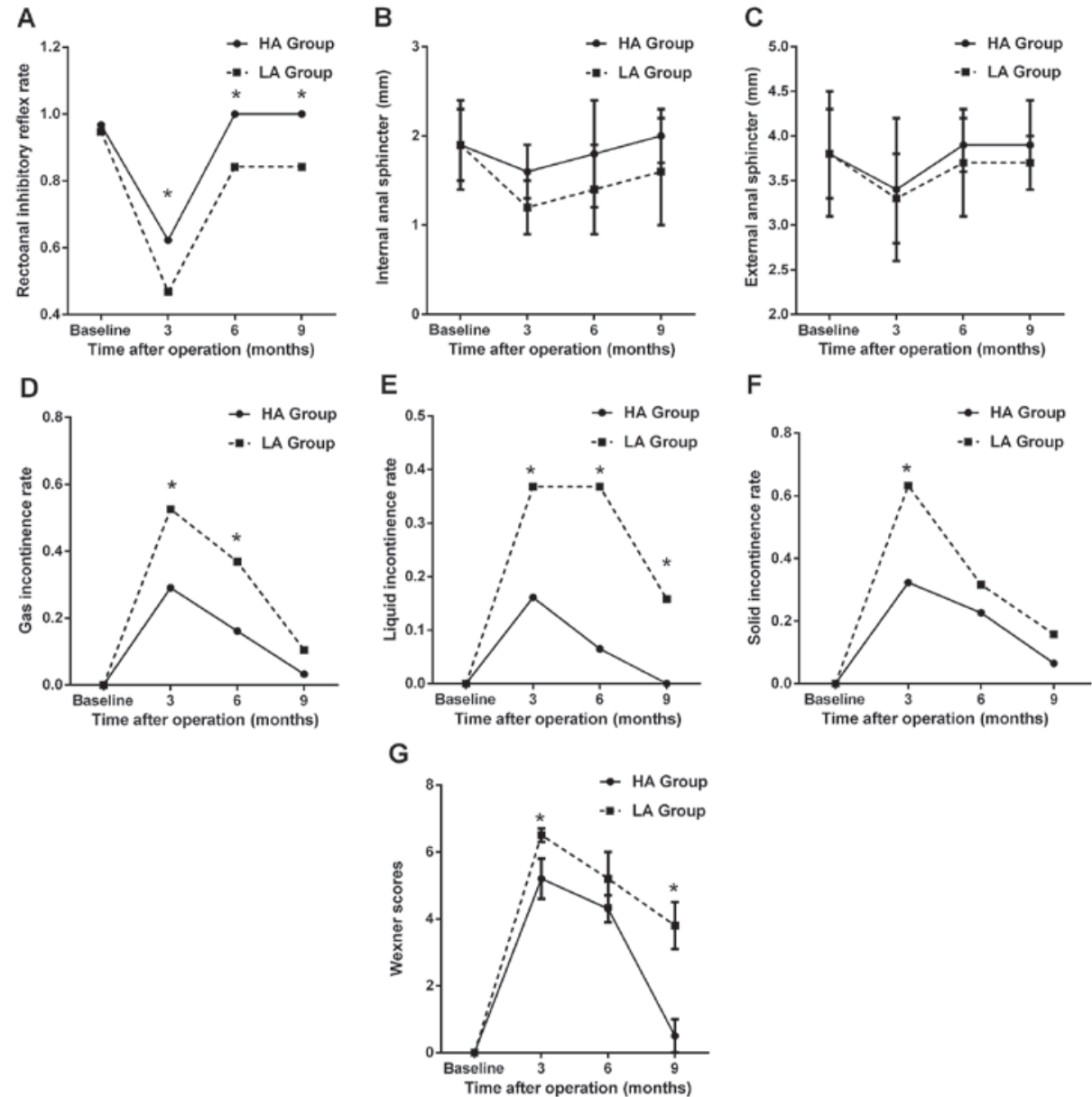

Figure 2. Changes in rectoanal inhibitory reflex, anal sphincter, incontinence and Wexner score. (A) Rectoanal inhibitory reflex rate. (B) Internal anal sphincter. (C) External anal sphincter. (D) Gas incontinence rate. (E) Liquid incontinence rate. (F) Solid incontinence rate. (G) Wexner score. Error bars indicate the standard error of the mean. ", statistically significant difference $(\mathrm{P}<0.05)$. HA, high anastomosis; LA, low anastomosis. 
A

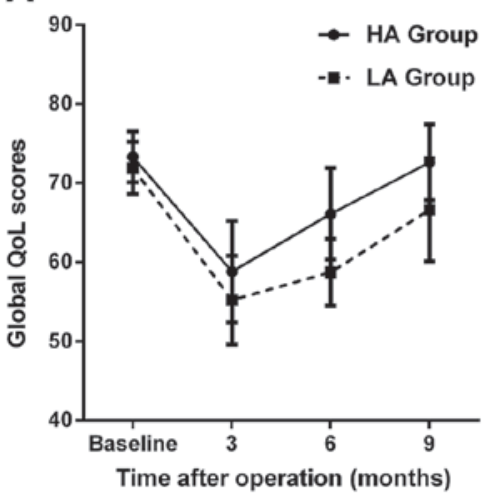

D

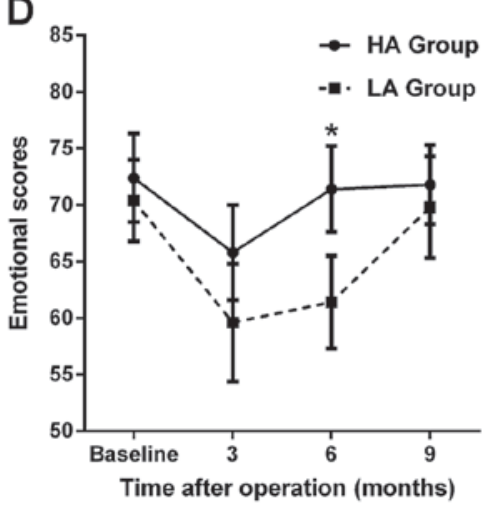

B
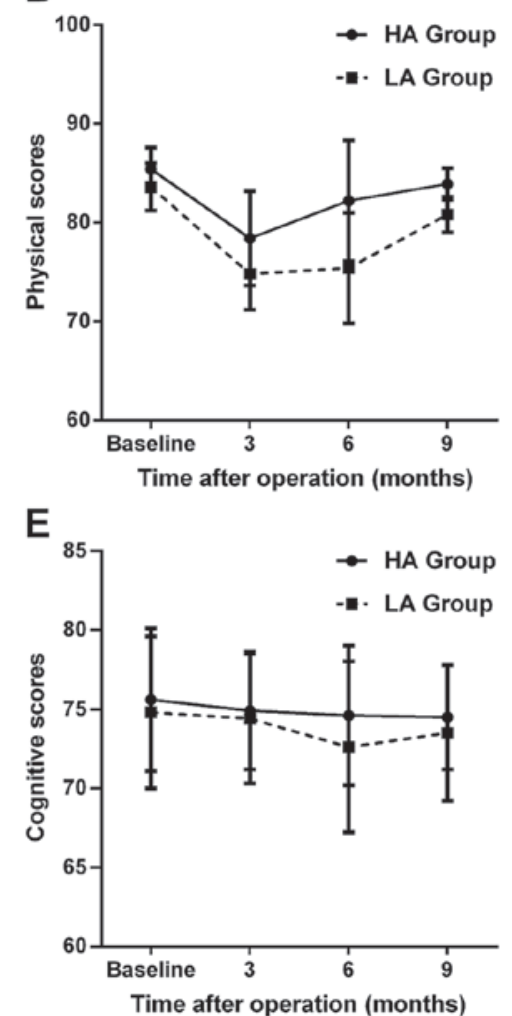

C
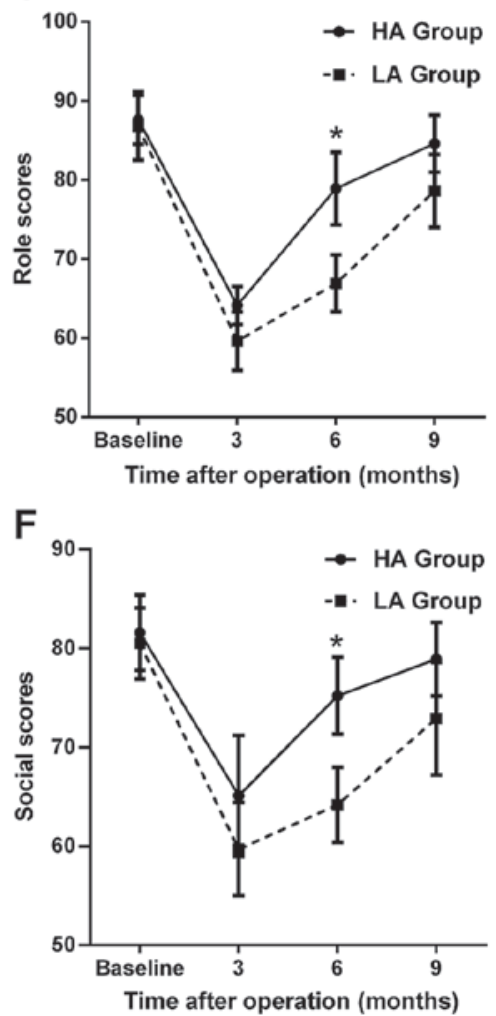

Figure 3. Longitudinal assessment of changes in QoL-C30 scores for various functioning scales. (A) Global QoL scores. (B) Physical scores. (C) Role scores. (D) Emotional scores. (E) Cognitive scores. (F) Social scores. Error bars indicate the standard error of the mean. *, statistically significant difference (P<0.05). HA, high anastomosis; LA, low anastomosis; QoL, quality of life.

(all $\mathrm{P}<0.05)$. Endoanal ultrasonography demonstrated rupture of IAS in 6 patients ( 1 in the HA and 5 in the LA group), with full integrity of EAS in all the patients. The changes in the thickness of IAS and EAS are shown in Fig. 2B and C. There was no difference in the thickness of IAS/EAS between the control and trial groups (all P>0.05, Table II). For the HA group, although a decreasing tendency was observed in the thickness of IAS and EAS at 3 months, the results were not statistically significant (all $\mathrm{P}>0.05$ ). Furthermore, at 6 and 9 months, the thickness of both the IAS and EAS had increased to the preoperative level (all $\mathrm{P}>0.05$ ). For the LA group, similar changes were only found in EAS; the thickness of the IAS was significantly reduced at 3 months postoperatively $(\mathrm{P}<0.05)$, with an insignificant increase at 6 and 9 months postoperatively (all $\mathrm{P}>0.05$ ).

Changes in incontinence and Wexner score. The changes in gas, liquid and solid incontinence rates are presented in Fig. 2D-F. The patients of the control and trial groups reported no incontinence prior to surgery. However, both subgroups experienced incontinence to different degrees at 3 months after LLAR (as shown in Fig. 2D-F). The liquid incontinence rate of the LA group was significantly higher compared with that of the HA group postoperatively (all $\mathrm{P}<0.05$ ), but gradually improved. The changes of the Wexner scores are demonstrated in Fig. 2G. For the HA group, the Wexner score exhibited an increase at 3 months postoperatively, but was decreased at 6 and 9 months, which was also observed in the LA group. Moreover, the Wexner scores of the HA group were significantly lower compared with those of the LA group at 3 and 9 months postoperatively (all $\mathrm{P}<0.05$ ).

Changes in QoL. The functioning scales of QoL-C30 are shown in Fig. 3. Compared with the preoperative level, the function scores of both groups decreased at 3 months postoperatively, but gradually increased by 9 months postoperatively, except for cognitive function (Fig. 3E). For the symptom scales of QoL-C30, as shown in Fig. 4, the reverse trends were observed, except for dyspnea (Fig. 4D) and constipation (Fig. 4G). On intergroup comparison, the HA group had a significantly better role, emotional and social function (Fig. 3C, D and F), and fewer diarrhea problems (Fig. 4H) at 6 months postoperatively compared with the LA group (all $\mathrm{P}<0.05$ ).

With regard to QoL-CR38 scores, as shown in Fig. 5, certain scales had changes similar to QoL-C30, such as sexual function (Fig. 5A), body image (Fig. 5B) and micturition problems (Fig. 5D). Chemotherapy-related side effects and gastrointestinal tract symptoms, which were increased prior to 6 months, had decreased to preoperative levels at 9 months postoperatively (Fig. 5E and F). Although the future perspective score was decreased at 3 months and slowly increased by 9 months postoperatively, it did not return to the preoperative level (Fig. 5C, $\mathrm{P}<0.05$ ). Moreover, there was a decreasing trend in the scores of defecation problems (Fig. 5G) and weight loss (Fig. 5H) postoperatively. On intergroup comparison, the HA group appeared to have a better sexual function (Fig. 5A) and fewer micturition problems (Fig. 5D) at 3 and 6 months postoperatively, with a better body image (Fig. 5B) and fewer 
A

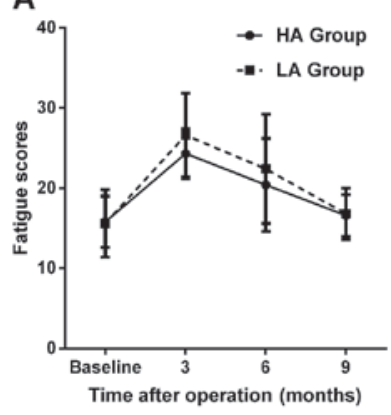

D

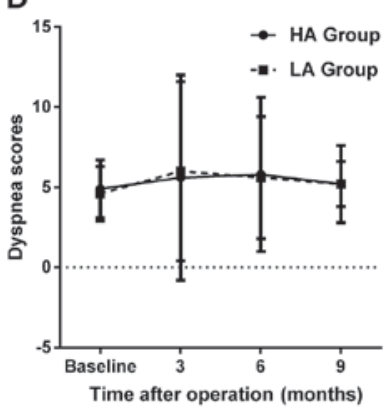

G

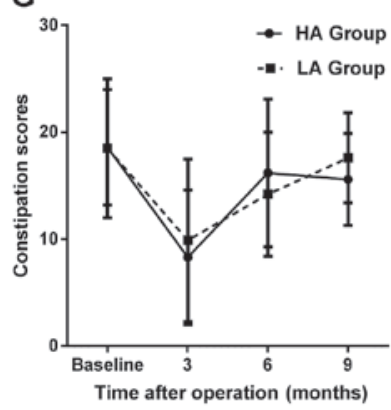

B

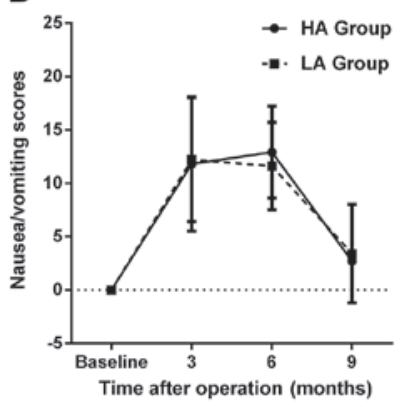

E

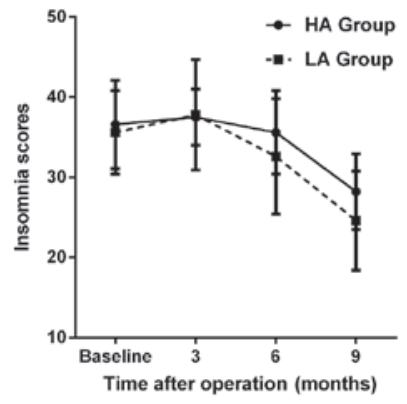

H

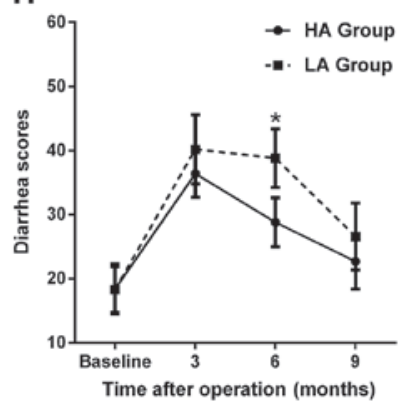

C

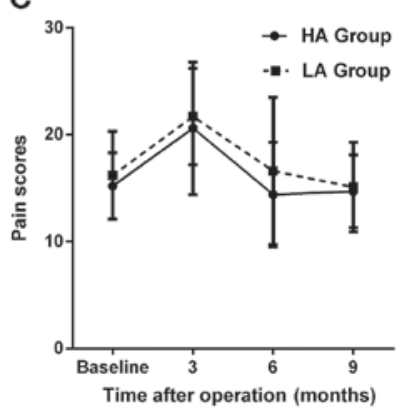

$F$

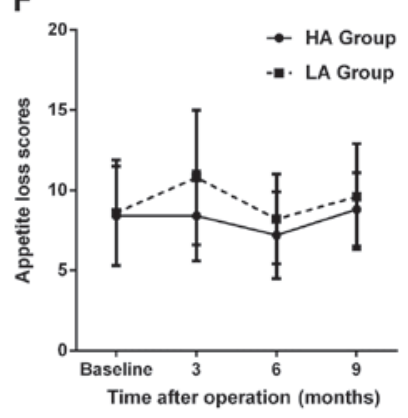

I

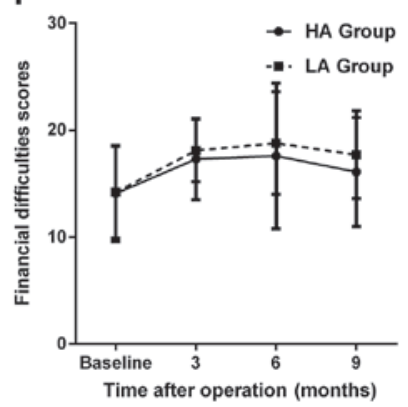

Figure 4. Longitudinal assessment of changes in QoL-C30 scores for various symptom scales. (A) Fatigue scores. (B) Nausea/vomiting scores. (C) Pain scores. (D) Dyspnea scores. (E) Insomnia scores. (F) Appetite loss scores. (G) Constipation scores. (H) Diarrhea scores. (I) Financial difficulties scores. ", statistically significant difference $(\mathrm{P}<0.05)$. HA, high anastomosis; LA, low anastomosis; QoL, quality of life.

chemotherapy-related side effects (Fig. 5E) and defecation problems (Fig. 5G) at 6 months compared with the LA group (all $\mathrm{P}<0.05$ ).

\section{Discussion}

In this study, routine anorectal manometry, questionnaire survey and radiological examination were first performed to investigate the functional outcome of patients following LLAR. In anorectal manometry, ARP is mainly dependent on IAS and associated with maintaining continence, while MSP is mainly dependent on EAS and associated with maintaining continence under stress. To the best of our knowledge, although no studies have investigated the manometric parameters following LLAR, several studies have demonstrated different manometric results after open LAR. Bittorf et al found the mean/maximum ARP to be significantly decreased, with unchanged MSP (23). Rasmussen et al reported that anal manometry was normal postoperatively, although the majority of the patients complained of poor functional results (24). A recent study indicated that both the ARP and MSP were significantly decreased at 6 and 12 months postoperatively (25). In our study, both the ARP and MSP were significantly reduced at 3 months postoperatively, but gradually improved to preoperative levels at 6 and 9 months. Since the anal sphincter plays a key role in maintaining pressure, endoanal ultrasonography examination was performed and the results displayed a similar trend in the thickness of IAS and EAS. This temporary injury of the anal sphincter was largely attributed to anal dilatation by stapling instruments during surgery, which finally resulted in the decreased pressures. With regard to the volume change of the rectum, a similar trend was also observed. However, all the volume parameters remained significantly lower compared with the preoperative values at 9 months after LLAR, largely due to the reconstruction of the rectum during the operation.

RAIR is defined as an important parasympathetic reflex controlling anorectal continence and involving a number of pelvic muscles, such as IAS and EAS. In this study, absent RAIR was found in 22 of the 50 patients at 3 months after LLAR. However, RAIR was gradually induced in the majority of the patients at $6(47 / 50)$ and $9(47 / 50)$ months after LLAR. This result was superior to the one demonstrated by Kakodkar et al, who found RAIR to be absent in all the patients at 6 months postoperatively, but present in half of the patients at 12 months after LAR (25). With regard to postoperative continence, a number of patients complained of gas, 
A

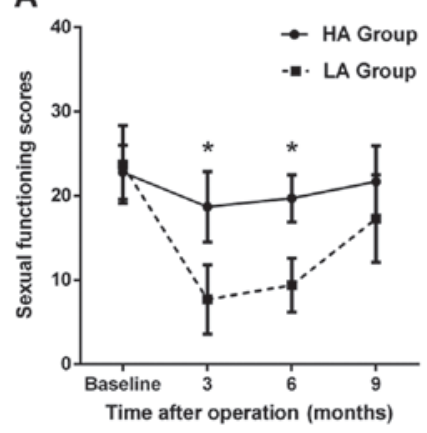

D

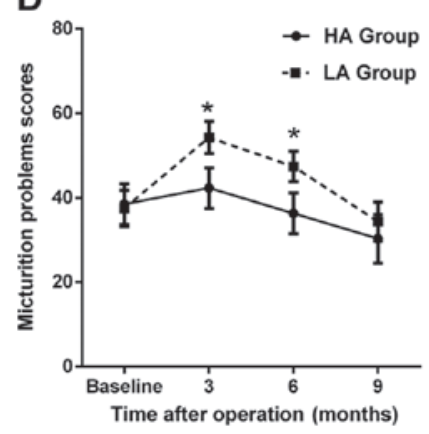

B

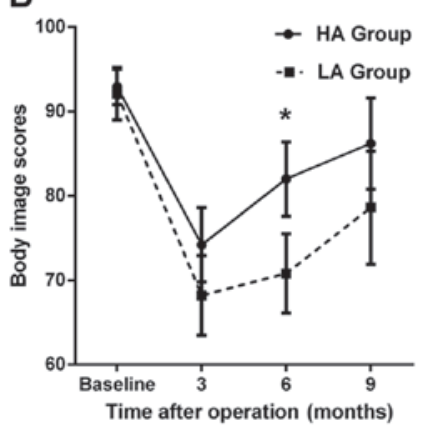

E

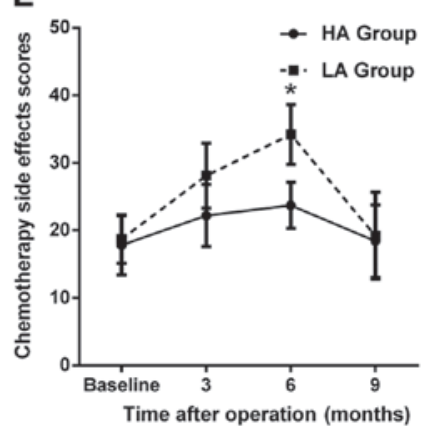

C

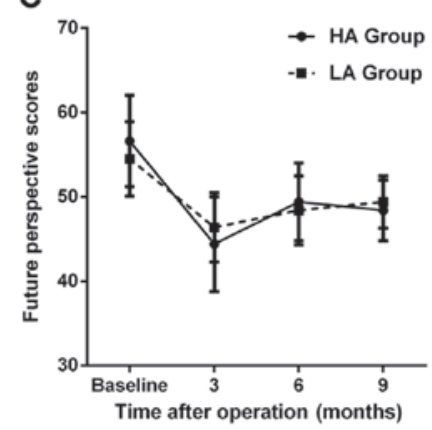

\section{$\mathbf{F}_{\text {. }}$}

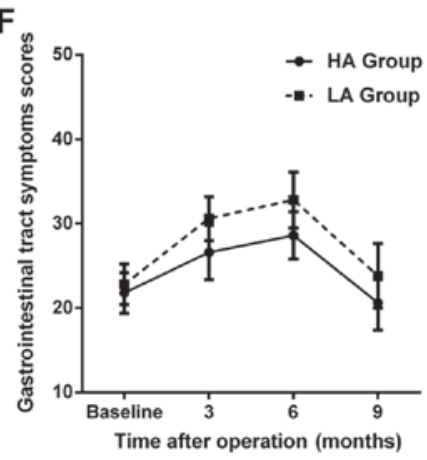

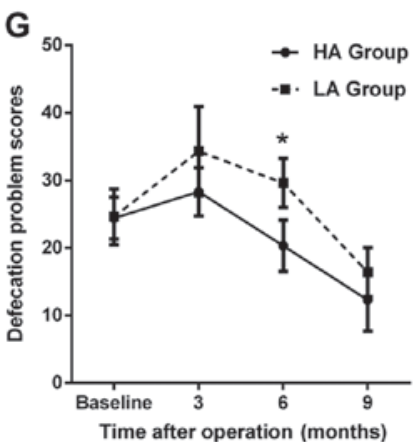

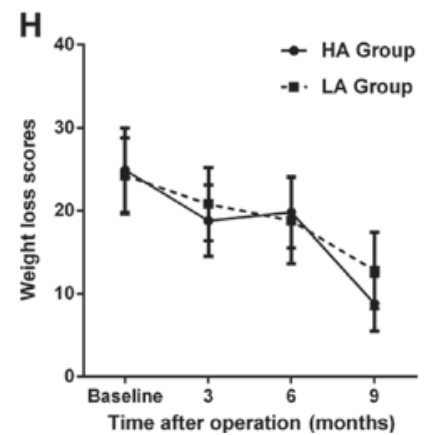

Figure 5. Longitudinal assessment of changes in QoL-CR38 scores for various functional and symptom scales. (A) Sexual functioning scores. (B) Body image scores. (C) Future perspective scores. (D) Micturition problems scores (E) Chemotherapy side effects scores. (F) Gastrointestinal tract symptoms scores. (G) Defecation problems scores. (H) Weight loss scores. ", statistically significant difference $(\mathrm{P}<0.05)$. HA, high anastomosis; LA, low anastomosis; QoL, quality of life.

liquid or solid incontinence at 3 months postoperatively, which had improved in the majority of the patients at 6 and 9 months postoperatively. This process was then confirmed by employing the Wexner score system, suggesting that LLAR may have a favorable outcome in terms of postoperative continence.

The patients were divided into subgroups to further investigate the effect of anastomotic height on anorectal function and QoL after LLAR. As a result, we found that the HA group had a higher ARP and MSP compared with the LA group at 3 and 6 months postoperatively. This difference was also observed in MTV, RAIR and IAS thickness postoperatively. Moreover, the LA group appeared to have a higher frequency of incontinence and higher Wexner scores compared with the HA group. Therefore, we hypothesized that anastomotic height may be a potential factor affecting functional outcome and QoL in Chinese patients following LLAR. This hypothesis has been indirectly supported by several studies regarding LAR. Montesani et al found that patients with anastomosis at $4-6 \mathrm{~cm}$ from the anal verge had poorer functional results compared with those with anastomosis at $6-8 \mathrm{~cm}$, despite the fact that the differences in most manometry parameters were statistically insignificant (26). In a recent questionnaire survey based on 381 cases, patients with low anastomosis were more likely to present with an increased frequency of defecation problems compared with those with a higher anastomosis (27). Additionally, a long-term study indicated that patients with anastomoses at $<4 \mathrm{~cm}$ from the anal verge had poorer functional results compared with those with anastomoses at $5-8 \mathrm{~cm}$ above the anal verge during the first 5 postoperative years (28). According to our surgical experience, this anastomotic effect on anorectal function may be explained as follows: i) Patients with low anastomosis usually undergo more extensive pelvic dissection during surgery compared with those with higher anastomosis, which may cause more injury to the IAS, finally resulting in poorer anorectal function; and ii) the nerve plexuses in the rectal wall play an important role in controlling the reflex of defecation and may be more easily injured in patients with low anastomosis.

QoL questionnaires are a subjective method for evaluating the impact of a disease and its related treatments on the patient's physical, psychological and social functioning (29). Previous studies have indicated that patients appeared to have 
better health-related QoL scores after LAR, when compared with those who underwent high anterior resection $(30,31)$. In the present study, we also observed that the HA group had more favorable QoL scores compared with the LA group postoperatively. These differences may be largely explained by the hypothesis that the LA group patients are more prone to develop what is referred to as the 'LAR syndrome', which comprises frequent defecation, fecal urgency and stool incontinence. Several previous studies have closely associated poor QoL with low anastomosis (32,33). However, no significant differences were observed in most functional or symptom scales between the two groups at 9 months postoperatively. This observation probably reflects the fact that LLAR may allow better preservation of the pelvic autonomic nerves through the magnified vision and less traumatic surgery (34). Although the future perspective score increased slowly from 3 to 9 months postoperatively, it did not return to the preoperative level, suggesting that high preoperative expectations for LLAR do not appear to translate into patient satisfaction within 9 months postoperatively, and long-term follow-up is required for further evaluation.

In summary, anorectal function following LLAR was found to be impaired, but improved over time. Patients with low anastomosis have poorer functional results and QoL compared with those with high anastomosis. Therefore, in terms of functional preservation, LLAR is generally acceptable for Chinese patients with rectal cancer, particularly for those with middle or high rectal cancer. A multicentric, long-term follow-up is required to further investigate the functional effect of LLAR in Chinese patients with rectal cancer.

\section{Acknowledgements}

The present study was supported by a grant from the Science and Technology Commission of Shanghai Municipality (no. 124119a720). The authors would like to thank Drs Jia-Yuan Peng and Qing-Chao Zhu (Department of Surgery, Sixth People's Hospital Affiliated to Shanghai Jiao Tong University) for their crucial assistance with our follow-up study.

\section{References}

1. Jemal A, Bray F, Center MM, Ferlay J, Ward E and Forman D: Global cancer statistics. CA Cancer J Clin 61: 69-90, 2011.

2. Bonjer HJ, Deijen CL, Abis GA, Cuesta MA, van der Pas MH, de Lange-de Klerk ES, Lacy AM, Bemelman WA, Andersson J, Angenete $\mathrm{E}$, et al: A randomized trial of laparoscopic versus open surgery for rectal cancer. N Engl J Med 372: 1324-1332, 2015.

3. Gu J and Chen N: Current status of rectal cancer treatment in China. Colorectal Dis 15: 1345-1350, 2013.

4. Hoerske C, Weber K, Goehl J, Hohenberger W and Merkel S: Long-term outcomes and quality of life after rectal carcinoma surgery. Br J Surg 97: 1295-1303, 2010.

5. Pachler J and Wille-Jørgensen P: Quality of life after rectal resection for cancer, with or without permanent colostomy. Cochrane Database Syst Rev 12: CD004323, 2012.

6. How P, Stelzner S, Branagan G, Bundy K, Chandrakumaran K, Heald RJ and Moran B: Comparative quality of life in patients following abdominoperineal excision and low anterior resection for low rectal cancer. Dis Colon Rectum 55: 400-406, 2012.

7. Kasparek MS, Hassan I, Cima RR, Larson DR, Gullerud RE and Wolff BG: Quality of life after coloanal anastomosis and abdominoperineal resection for distal rectal cancers: Sphincter preservation vs quality of life. Colorectal Dis 13: 872-877, 2011.
8. van Leersum N, Martijnse I, den Dulk M, Kolfschoten N, Le Cessie S, van de Velde C, Tollenaar R, Wouters M and Rutten HJ: Differences in circumferential resection margin involvement after abdominoperineal excision and low anterior resection no longer significant. Ann Surg 259: 1150-1155, 2014.

9. Veldkamp R, Kuhry E, Hop WC, Jeekel J, Kazemier G, Bonjer HJ, Haglind E, Påhlman L, Cuesta MA, Msika S, et al: Laparoscopic surgery versus open surgery for colon cancer: Short-term outcomes of a randomised trial. Lancet Oncol 6: 477-484, 2005.

10. Ng SS, Leung WW, Wong CY, Hon SS, Mak TW, Ngo DK and Lee JF: Quality of life after laparoscopic vs open sphincter-preserving resection for rectal cancer. World J Gastroenterol 19: 4764-4773, 2013.

11. Chi P, Huang SH, Lin HM, Lu XR, Huang Y, Jiang WZ, Xu ZB, Chen ZF, Sun YW and Ye DX: Laparoscopic transabdominal approach partial intersphincteric resection for low rectal cancer: Surgical feasibility and intermediate-term outcome. Ann Surg Oncol 22: 944-951, 2015.

12. Kneist W, Kauff DW, Juhre V, Hoffmann KP and Lang H: Is intraoperative neuromonitoring associated with better functional outcome in patients undergoing open TME? results of a case-control study. Eur J Surg Oncol 39: 994-999, 2013.

13. Kauff DW, Koch KP, Somerlik KH, Hoffmann KP, Lang H and Kneist W: Evaluation of two-dimensional intraoperative neuromonitoring for predicting urinary and anorectal function after rectal cancer surgery. Int J Colorectal Dis 28: 659-664, 2013.

14. Maris A, Penninckx F, Devreese AM, Staes F, Moons P, Van Cutsem E, Haustermans K and D'Hoore A: Persisting anorectal dysfunction after rectal cancer surgery. Colorectal Dis 15: e672-e679, 2013

15. Kye BH, Kim HJ, Kim JG, Kim SH, Shim BY, Lee NS and Cho HM: Short-term effects of neoadjuvant chemoradiation therapy on anorectal function in rectal cancer patients: A pilot study. Radiat Oncol 8: 203, 2013.

16. Pietsch AP, Fietkau R, Klautke G, Foitzik T and Klar E: Effect of neoadjuvant chemoradiation on postoperative fecal continence and anal sphincter function in rectal cancer patients. Int $\mathrm{J}$ Colorectal Dis 22: 1311-1317, 2007.

17. Gong X, Jin Z and Zheng Q: Anorectal function after partial intersphincteric resection in ultra-low rectal cancer. Colorectal Dis 14: e802-e806, 2012.

18. Jin Z, Yin L, Xue L, Lin M and Zheng Q: Anorectal functional results after transanal endoscopic microsurgery in benign and early malignant tumors. World J Surg 34: 1128-1132, 2010.

19. Zhang HW, Han XD, Wang Y, Zhang P and Jin ZM: Anorectal functional outcome after repeated transanal endoscopic microsurgery. World J Gastroenterol 18: 5807-5811, 2012.

20. Zhu QL, Feng B, Lu AG, Wang ML, Hu WG, Li JW, Mao ZH and Zheng MH: Laparoscopic low anterior resection for rectal carcinoma: Complications and management in 132 consecutive patients. World J Gastroenterol 16: 4605-4610, 2010.

21. Kosmidis C, Efthimiadis C, Anthimidis G, Grigoriou M, Fotiadis P, Vasiliadou K, Mekras D, Ioannidou G, Baka S and Basdanis G: Laparoscopic low anterior resection for early rectal cancer. Tech Coloproctol 15 (Suppl 1): S75-S77, 2011.

22. Konanz J, Herrle F, Weiss C, Post S and Kienle P: Quality of life of patients after low anterior, intersphincteric and abdominoperineal resection for rectal cancer-a matched-pair analysis. Int J Colorectal Dis 28: 679-688, 2013.

23. Bittorf B, Stadelmaier U, Göhl J, Hohenberger W and Matzel KE: Functional outcome after intersphincteric resection of the rectum with coloanal anastomosis in low rectal cancer. Eur J Surg Oncol 30: 260-265, 2004.

24. Rasmussen OO, Petersen IK and Christiansen J: Anorectal function following low anterior resection. Colorectal Dis 5: 258-261, 2003.

25. Kakodkar R, Gupta S and Nundy S: Low anterior resection with total mesorectal excision for rectal cancer: Functional assessment and factors affecting outcome. Colorectal Dis 8: 650-656, 2006.

26. Montesani C, Pronio A, Santella S, Boschetto A, Aguzzi D, Pirozzi R, D'Amato A and Vestri A: Rectal cancer surgery with sphincter preservation: Functional results related to the level of anastomosis. clinical and instrumental study. Hepatogastroenterology 51: 718-721, 2004.

27. Knowles G, Haigh R, McLean C, Phillips HA, Dunlop MG and Din FV: Long term effect of surgery and radiotherapy for colorectal cancer on defecatory function and quality of life. Eur J Oncol Nurs 17: 570-577, 2013. 
28. Hida J, Yoshifuji T, Matsuzaki T, Hattori T, Ueda K, Ishimaru E, Tokoro T, Yasutomi M, Shiozaki H and Okuno K: Long-term functional changes after low anterior resection for rectal cancer compared between a colonic J-pouch and a straight anastomosis. Hepatogastroenterology 54: 407-413, 2007.

29. Outcomes of cancer treatment for technology assessment and cancer treatment guidelines. American society of clinical oncology. J Clin Oncol 14: 671-679, 1996.

30. Grumann MM, Noack EM, Hoffmann IA and Schlag PM: Comparison of quality of life in patients undergoing abdominoperineal extirpation or anterior resection for rectal cancer. Ann Surg 233: 149-156, 2001.

31. Engel J, Kerr J, Schlesinger-Raab A, Eckel R, Sauer H and Hölzel D: Quality of life in rectal cancer patients: A four-year prospective study. Ann Surg 238: 203-213, 2003.
32. Hassan I, Larson DW, Cima RR, Gaw JU, Chua HK, Hahnloser D, Stulak JM, O'Byrne MM, Larson DR, Wolff BG and Pemberton JH: Long-term functional and quality of life outcomes after coloanal anastomosis for distal rectal cancer. Dis Colon Rectum 49: 1266-1274, 2006.

33. Ziv Y, Zbar A, Bar-Shavit Y and Igov I: Low anterior resection syndrome (LARS): Cause and effect and reconstructive considerations. Tech Coloproctol 17: 151-162, 2013.

34. Scheele J, Lemke J, Meier M, Sander S, Henne-Bruns D and Kornmann M: Quality of life after sphincter-preserving rectal cancer resection. Clin Colorectal Cancer 14: e33-e40, 2015. 